\title{
The Wind of Change: Uncomplicated Diverticulitis
}

\author{
Hungdai Kim \\ Department of Surgery, Gastrointestinal Cancer Center, Kangbuk Samsung Hospital, Seoul, Korea
}

\section{See Article on Page 52-56}

Among the wide spectrum of acute diverticulitis, uncomplicated diverticulitis (UD) is defined as a localized inflammation or phlegmon of colonic diverticula in the absence of complications such as abscess, perforation, fistula, obstruction, and bleeding. Acute diverticulitis calls for a differentiated approach to various stages of the disease process. According to guidelines from a related international academic society, the use of antibiotics, either intravenous or oral, is the nub of management in patients with UD. Recently, evidence has accumulated that the majority of these patients successfully respond to outpatient management or oral antibiotic use [1]. Outpatient management of patients with UD is also less expensive for the health care system. A randomized controlled trial from Sweden found that antibiotic use for the treatment of UD neither accelerated recovery nor prevented complications or recurrence [2].

Scarpa et al. [3] have compared the treatment outcomes for patients with UD for different routes of antibiotic administration. Patients were divided into two groups: one received antibiotics entirely via an intravenous route while the other received antibiotics via an intravenous + oral route. According to the investigators, the use of intravenous antibiotics for less than five days did not increase the recurrence of uncomplicated colonic diverticulitis. This finding indicates that patients might be able to avoid an unnecessary admission. However, as the authors mentioned, regardless of the route, intravenous or intravenous + oral, all patients received antibiotics for the same period. Therefore, huge room for investigations on the use of antibiotics still exists.

In 2014, The American Society of Colon and Rectal Surgeons revised its statement about 'Practice parameters for sigmoid di-

Correspondence to: Hungdai Kim, M.D.

Department of Surgery, Gastrointestinal Cancer Center, Kangbuk Samsung

Hospital, 29 Saemunan-ro, Jongno-gu, Seoul 110-746, Korea

Tel: +82-2-2001-8541, Fax: +82-2-2001-2131

E-mail: hungdai.kim@samsung.com

(c) 2015 The Korean Society of Coloproctology

This is an open-access article distributed under the terms of the Creative Commons Attribution NonCommercial License (http://creativecommons.org/licenses/by-nc/3.0) which permits unrestricted noncommercial use, distribution, and reproduction in any medium, provided the original work is properly cited. verticulitis' that was previously published in 2007 [4]. The most remarkable difference about UD management between the 2007 and the 2014 versions is the introduction of oral antibiotic use as an initial treatment for clinically-stable patients with UD. That the newly emerging trend for UD management is moving to an outpatient setting is becoming more and more obvious. The key to treatment success depends very much on an accurate diagnosis of the stage of acute diverticulitis.

The diagnosis of diverticular disease can be made by using a variety of tests, and the accuracy of imaging studies greatly affects the outcome of treatment for UD. Nowadays, computed tomography (CT) scanning is the most appropriate imaging modality to confirm suspected diverticulitis. However, the accuracy of predicting Hinchey classification by using CT scanning is not very high. Gielens et al. [5] reported in their study that the sensitivity and the specificity of CT scans for Hinchey 1 patients were $76 \%$, and $86 \%$, respectively. Even in $42 \%$ of the cases, Hinchey 3 perforated diverticulitis was falsely classified as Hinchey 1 or 2 . Therefore, before starting the use of antibiotics, a decision making process should be discreetly and conservatively set up to prevent treatment failure. In future studies, investigators should focus on the area of accurate diagnosis of patients for current treatment options.

Overall, we may certainly infer that a lesser aggressive treatment seems to be possible for the management of selected patients with UD. However, a considerable number of open questions still remain: for example, the efficacy of treatment with oral antibiotics only, the adequate duration of antibiotic treatment, the feasibility of not using antibiotics, and the role of probiotics and/or anti-inflammatory drugs. Further randomized clinical trials to establish an optimal strategy for treating uncomplicated colonic diverticulitis are encouraged.

\section{CONFLICT OF INTEREST}

No potential conflict of interest relevant to this article was reported.

\section{REFERENCES}

1. Jackson JD, Hammond T. Systematic review: outpatient manage- 
ment of acute uncomplicated diverticulitis. Int J Colorectal Dis 2014;29:775-81.

2. Chabok A, Pahlman L, Hjern F, Haapaniemi S, Smedh K; AVOD Study Group. Randomized clinical trial of antibiotics in acute uncomplicated diverticulitis. Br J Surg 2012;99:532-9.

3. Scarpa CR, Buchs NC, Poncet A, Konrad-Mugnier B, Gervaz P, Morel P, et al. Short-term intravenous antibiotic treatment in uncomplicated diverticulitis does not increase the risk of recurrence compared to long-term treatment. Ann Coloproctol 2015;31:52-6.

4. Feingold D, Steele SR, Lee S, Kaiser A, Boushey R, Buie WD, et al. Practice parameters for the treatment of sigmoid diverticulitis. Dis Colon Rectum 2014;57:284-94.

5. Gielens MP, Mulder IM, van der Harst E, Gosselink MP, Kraal KJ, Teng HT, et al. Preoperative staging of perforated diverticulitis by computed tomography scanning. Tech Coloproctol 2012;16:363-8. 